\title{
Les comptines à caractère énigmatique dans le conte de tradition orale
}

\author{
Anna Angelopoulos \\ Université Paris III Sorbonne Nouvelle \\ angelopoulos.anna@gmail.com
}

\section{RÉSUMÉ}

Dans les contes de tradition orale, il existe parfois des formulettes racontant le parcours du héros de façon sommaire et énigmatique. Ces énoncés viennent en lieu et place d'un agir du personnage principal et révèlent son identité cachée en cas de travestissement ou de métamorphose. Ainsi le héros ou l'héroïne est reconnu(e) par toutes les personnes mises en scène et par l'assistance.

L'auteur prend deux exemples, afin d'illustrer son propos; le conte merveilleux "L'enfant artificiel » (705) et le conte-nouvelle "Turandot» (85I). Dans les versions présentées de ces contes-types, l'usage de comptines brèves sert à dénouer une intrigue dramatique qui recouvre le parcours secret du héros vers la rencontre ou les retrouvailles amoureuses.

MOTS CLÉS

conte, métamorphose, identité, ATU 705, ATU 85 I

RESUM

En els contes de tradició oral, de vegades hi ha rimes que relaten el viatge de l'heroi de manera breu i enigmàtica. Aquests enunciats hi apareixen quan el protagonista actua, $i$ en revelen la identitat amagada en cas de transvestisme o de metamorfosi. Així, l'heroi o l'heroïna és reconegut o reconeguda per totes les persones en escena, així com pel públic.

En prendrem dos exemples, per tal d'il.lustrar els nostres comentaris; la rondalla meravellosa "Nascut de la cama de son pare» (ATU 705) i la rondalla d'enginy "L'enigma del mandrós» (ATU 85I). En aquests relats, l'ús de cançons infantils breus serveix per desenredar una intriga dramàtica que recobreix el recorregut secret de l'heroi cap al retrobament o les retrobades amoroses.

PARAULES CLAU

rondalla, metamorfosi, identitat, ATU 705, ATU 85 I 
Anna Angelopoulos

\begin{abstract}
Tales from the oral tradition sometimes feature rhymes that describe the hero's journey in a brief and enigmatic manner. These rhymes appear when the protagonist acts and reveal his or her hidden identity in cases of transvestism or metamorphosis. In this way the hero or heroine is recognized by every person in the scene and by the audience.

I will take two examples to illustrate the points I wish to make. The first is the tale of magic "Born from the leg of his father" (ATU 705) and the realistic tale "The enigma of the lazy man" (ATU 85I). In these tales, the use of short children's songs serves to untangle the dramatic intrigue that hides the secret journey of the hero towards the amorous reencounter.
\end{abstract}

KEYWORDS

folktale, metamorphosis, identity, ATU 705, ATU 851 
LES COMPTINES BRÈVES, chantées ou parlées à la fin de certains contes, lors du dénouement de l'intrigue, appellent à la mise en récit par le héros de son histoire, de sa traversée. Ces formulettes se trouvent tant dans les contes merveilleux que dans les contes-nouvelles ou les contes facétieux. J'ai donc choisi de présenter l'exemple d'un conte merveilleux (ATU 705) qui utilise une comptine énigmatique pour le dénouement de son intrigue, ainsi qu'un conte-nouvelle (ATU 85I) qui aboutit à la formulation d'une devinette dont la solution est impérative pour que l'histoire puisse se conclure heureusement. Dans les deux cas, un personnage du conte devra se faire deviner par un autre dans un rapport d'adresse amoureuse.

Je présenterai d'abord une version grecque du conte-nouvelle connu comme Turandot (ATU 85I), intitulée « l'énigme du paresseux ». Ce conte fait partie d'un groupe spécial de contes-devinettes selon Joan Amades (Amades I960: 199-223). Dans son article, il classe les histoires contenant des énigmes dans quatre groupes utilisant des devinettes plus ou moins narratives qui servent à des causes diverses, telles que par exemple:

- Epouser la princesse qui ne peut résoudre l'énigme,

- Libérer un membre de sa famille condamné ou prisonnier, grâce à une énigme que son geôlier ne pourra résoudre,

- Gagner sa vie en l'exploitant sous la forme d'une énigme,

- Sauver enfin sa vie (ou la vie d'un autre) en échange d'une énigme insoluble.

Dans tous les cas, ces comptines énigmatiques sont présentées au point culminant de l'histoire et résument de façon succincte le parcours initiatique du protagoniste. Telles le refrain d'une chanson, ces paroles reprennent, dans un langage figuré, tous les moments forts de la narration. Elles peuvent ainsi servir d'aide mnémotechnique pour le conteur; mais, elles peuvent aussi marquer le moment dans le récit où s'opère pour le personnage principal le passage de l'énumération de ses épreuves à la symbolisation de sa traversée.

Afin d'éclaircir ce point, notons que le héros du conte, après avoir survécu à des aventures extrêmes, se trouve parfois sollicité pour en faire le récit. Il prend alors la parole dans un langage codé, et parle par des métaphores à l'assistance. Ce point est décisif, car le héros à devenir parvient ainsi à l'assomption de son identité, il parle en tant que sujet à la fin du conte, ce qui conduit au dénouement de l'intrigue. Dans le cas du conte-nouvelle présenté ici, connu comme Turandot (ATU 85I), le personnage principal, un jeune paresseux, possède en fin de parcours un secret qu'il peut laisser deviner aux autres. Il leur apporte une énigme à résoudre, s'enrichit d'elle, et en fait une acquisition. Il s'agit bien du secret de sa propre vie qui devient en l'occurrence un métalangage. 
Ce garçon paresseux et niais, en début du récit, figure proche de Jean le Sot du conte facétieux, est un héros agi tout le long de l'intrigue par des forces qui le dépassent. Tout d'abord, par la volonté de sa mère de se débarrasser de lui; elle lui donne un pain empoisonné pour la route; son chien le mange et en meurt. Le garçon paresseux lance ensuite des flèches contre un aigle qui se saisit du cadavre de son chien, mais il tue par erreur une jument; de son cadavre, il retire un poulain et fait rôtir la chair de sa mère la jument, en faisant du feu avec des livres d'église. Il boit de l'eau du bénitier; il traverse un pont et le voit être englouti par un torrent qui emporte également tout un troupeau de chèvres.

A la fin de son parcours, le garçon paresseux arrive dans un pays où le roi annonce qu'il donnera la main de sa fille à celui qui posera une énigme insoluble, qu'on ne trouve pas dans les livres. Il raconte alors son histoire et gagne le pari. Ainsi, comme tant d'autres, ce héros advient à lui-même lorsqu'il parvient à se raconter. Il a vécu les choses par le corps jusqu'à présent, mais en les formulant par des mots, dans des phrases, il devient enfin le sujet de son histoire. D'ailleurs, le conteur lui passe la parole, il interrompt le récit à la troisième personne afin que l'assistance ait droit au récit énigmatique du héros « en direct » à la première personne. Dans les deux cas, celui du conte merveilleux et du conte-nouvelle, le personnage principal dit: « je » quand il énonce son témoignage de survivant dans un langage sibyllin et poétique.

Le jeune paresseux ne peut plus rester dans l'ignorance de sa propre altérité, du moment que sa mère, son seul lien au monde, a tenté de l'empoisonner. Ce fils unique de veuve esseulée est ainsi arraché à sa mère, agi par sa tentative de meurtre qui lui est bien adressée, mais qui tue finalement sa petite chienne, son unique compagnie de route, morte à sa place. La rupture violente de la relation duelle, mère veuve/fils paresseux, libère le jeune homme du monde imaginaire où il est enfermé et le rend attentif à son entourage; rupture qui le pousse à sortir de son état de «marge du symbolique » (Chardenet 2oIo). Le garçon niais est forcé de prendre son autonomie de survie. Il va connaître une succession de morts par empoisonnement et il va tuer des animaux quasiment sans y penser. Il est pris dans les évènements tumultueux de son parcours et avance sans s'en rendre compte vers le devenir homme adulte, vers la symbolisation. Il apprend bon gré mal gré à faire la différence entre ce qui est vivant et ce qui est mort, car il se voit lui-même tantôt vivant et tantôt mort. Il s'identifie à ces animaux tués, rencontrés sur son chemin, il en tue d'autres en donnant en même temps la vie à son insu, sans le vouloir. En regardant la jument morte éventrée et le poulain nouveau-né trotter déjà sur ses pattes, il repère le rapport entre naissance /passage matériel/vie/ et naissance à la vie sans passage matériel. Il se libère ainsi du fantasme fusionnel charnel avec le corps maternel. Il peut fantasmer que sa mère tueuse est morte à sa place et qu'il est désormais seul en vie. Il suit sa route d'expériences insolites extrêmes et arrive à présenter à la princesse une énigme insoluble. Son destin n'est pas régressif car il s'éloigne physiquement et psychiquement du corps à corps avec sa mère et avance vers la rencontre de l'autre. En arrivant devant le pont, emporté par le torrent, il réalise la force du destin, son impuissance devant les catastrophes naturelles et son état de rescapé dans la vie.

A la question «que veut la princesse qui cherche à épouser l'homme qui lui pose une énigme insoluble? » nous pouvons donner un élément de réponse. Elle cherche un jeune homme qui détient un savoir qui la dépasse. Attachée à son 
père, elle acceptera seulement l'homme qui pourra l'écarter de son lien indéfectible paternel. En effet, le garçon paresseux vient de loin; il a pu voler son savoir à son propre destin, il a suivi son chemin en avançant contre son gré. Le héros du conte-nouvelle advient comme sujet grâce à son expérience de passage obligé par la mort à la vie, grâce à sa nouvelle naissance de rescapé. Il obtient le pouvoir de plaire en détenteur d'énigmes subtiles qui ne sont autres que le récit de son propre destin. Il devient sujet au moment de l'acquisition de sa capacité narrative. Il est alors intéressant, aimable, mariable à la princesse. Celle-ci tente d'ailleurs de lui arracher la solution de son énigme la nuit, en couchant avec lui, mais cela est déjà l'intrigue d'un autre conte-nouvelle («Les marques de la princesse », ATU 850) à déchiffrer une prochaine fois.

Passons au conte merveilleux à présent. Il s'agit du conte ATU 705, The Artificial Child (Ma mère fut une jambe). L'héroïne est un personnage mythique, dont la naissance fait penser à celle d'Athéna qui est sortie de la tête paternelle. On pense également à Dionysos, sorti de la cuisse de Zeus. Il s'agit de motifs de naissances merveilleuses dans le conte populaire, d'images du corps divin en morceaux.

L'héroïne du conte est conçue dans la jambe (la tête) d'un homme (un prêtre), lequel avala par erreur une pomme destinée à féconder sa femme stérile. Neuf mois plus tard, dans la forêt, le prêtre accouche d'une petite fille, grâce à une épine qui lui déchire la peau de la jambe et rentre chez lui sans s'apercevoir de la naissance de son enfant.

La petite fille est emportée par un aigle dans son nid et élevée par lui. Elle vit sur un arbre sans fréquenter les humains, jusqu'au jour où le roi la voit et en tombe amoureux. Pour la faire descendre de l'arbre, il est aidé par une vieille femme, qui fait semblant d'être sourde et incite la jeune fille à descendre plus bas. Le roi l'attrape alors et l'épouse.

Plus tard, il part en guerre en confiant sa femme à sa mère qui répudie l'héroïne du palais et l'envoie garder les oies. Dans certaines versions, lui ayant tondu la tête, la belle-mère jette l'héroïne dans un puits, d'où elle chante ses aventures. Pendant son errance, la jeune femme bannie retrouve l'aigle et revit avec lui. Elle raconte son histoire ou bien elle la chante lors d'une veillée. Le roi malheureux qui se promène dans la forêt reconnaît alors, dans la chanson, la voix et le récit de sa femme. Reconnaissance des époux, punition cruelle de la marâtre. L'héroïne ramène l'aigle (sa mère adoptive) au palais et ils vivent tous heureux ensemble.

Notons une différence entre les versions, scandinaves pour la plupart, et grecques de ce conte. En Grèce, la fille sortie de la tête ou de la jambe paternelle ne fait pas d'enfants qui lui seront enlevés par la suite, selon le schéma de la femme persécutée qu'on connaît depuis plusieurs contes merveilleux (« La fille sans mains », « Blanche-Neige », « Les trois enfants d'or »). Le reste des épisodes du récit de « la femme persécutée » y sont présents toujours dans l'ordre: bannissement, errance dans la forêt, déchéance, révélation de la vérité et réunion des époux. Mais, en général, le motif de l'enfantement manque pour l'héroïne du conte «L'enfant artificiel » (à l'exception de 2 versions sur 55).

L'héroïne reste donc toujours une enfant artificielle qui subit des épreuves d'humanisation, car elle n'a pas fait le passage par les voies utérines pour naître. La jambe du pope fonctionne comme un utérus artificiel qui la met juste au monde. Notons que le pope continue son chemin et rentre chez lui sans se douter de cette naissance. Selon certaines versions, le prêtre s'évanouit en la mettant au monde, 
selon d'autres, il est présent, il couvre le nourrisson avec sa chemise, mais il laisse sa progéniture dans la forêt et rentre chez lui sans en parler à sa femme. Le rapt par l'aigle s'apparente alors à une intervention divine de sauvetage. Cet enfant né du père, sans mère, est fait pour grandir dans les hauteurs du ciel, dans le nid de l'aigle. L'héroïne devient ainsi « la fille de l'aigle », patronne des oiseaux, espèce maternelle sauvage qui lui apprend à parler sa langue et à se méfier des humains. Elle reste en quelque sorte l'enfant sauvage d'une mère-oiseau jusqu'à la fin. Il s'agit, dans le contexte du conte, d'un mythe de création par un père d'une fille qui, telle Athéna, demeure étrangère à la procréation et à la progéniture.

La jeune fille subite néanmoins des épreuves d'humanisation, paradoxalement, avec des mauvaises figures féminines, d'abord la vieille rusée qui la fait descendre de l'arbre et ensuite la mauvaise belle-mère qui la répudie. L'héroïne doit sacrifier sa part mythique à l'humain. Se faire reconnaître par la mère de l'époux s'avère impossible. Obtenir le statut de belle-fille est pour elle un véritable rite de passage qui l'envoie aux enfers. Cependant sa relation à la marâtre sera constituante pour sa féminité au terme de ses aventures.

Comme pour d'autres héroïnes du conte appartenant au cortège des épouses bannies, l'enjeu est pour elle de rassembler les deux images extrêmes de sa personne, à la fois fille de l'oiseau céleste et gardeuse d'oies, tombée de haut dans la basse-cour. "Toi, fille d'aigle sans famille aucune, tu n'est pas faite pour être reine et femme de roi ! Car la reine c'est moi». Ce qui cause le rejet de la jeune épouse c'est son illégitimité: bâtarde, de parents humains inconnus, elle n'est bonne qu'à être détrônée de sa place, qu'à garder les oies (Cendrillon des champs!) La voilà tombée au plus bas. Cet enfer dure trois ans, à rester cachée dans la pénombre sans parler, à pleurer parmi les oies et les serviteurs. Sa situation énigmatique qui l'a propulsée du plus haut au plus bas l'isole en elle même.

Au bout de trois ans, un soir, à l'heure des contes, la gardeuse d'oie toujours silencieuse est sollicitée pour raconter un conte. Elle se met à chanter une énigme ${ }^{\mathrm{I}}$, l'histoire de sa vie comportant la partie manquante de sa préhistoire d'avant sa mère aigle. Autrement dit, elle lève l'amnésie sur ses parents humains, sur sa conception : la pomme, sa gestation dans la jambe de son père, l'épine qui en déchirant la jambe enflée de son père l'a fait naître. "Conte je suis », dit-elle. En effet, elle est maintenant l'auteur de la narration complète de sa propre vie, en restitue la partie manquante qui faisait d'elle une enfant sans parents humains, tombée du ciel. Son conte est chanté, comme revenu avec le souvenir d'une chanson lointaine.

Elle est elle-même cette histoire en énigme dont la narration prend tout son sens en étant reconnue par le prince, ce qui lui redonne son statut de femme royale à part entière.

Cette énigme chantée ramène, par le chant, le souvenir de la préhistoire occultée de l'héroïne et lève l'amnésie sur sa supposée illégitimité humaine. Elle libère des affects infantiles prêts à ressurgir dans sa détresse. Cette énigme se récite en vers et ne peut prendre sens qu'entendu par son époux amoureux.

I. Ce sont souvent des oiseaux qui chantent les énigmes qui révèlent l'identité cachée du sujet. Exemples, Le Genevrier (ATU 720), Blanche-Neige(ATU 709), L'oiseau de vérité (ATU 707), et bien d'autres. Dans quelques versions de ce conte, l'héroïne est transformée en oiseau car la marâtre lui enfonce une épingle dans le crâne. 
En même temps, le conteur nous fait part de son idée sur le conte par ce poème qu'il met dans la bouche de l'héroïne:

Un conte à raconter?

Conte je suis!

Une femme de prêtre me désira

Une pomme me conçut

Un prêtre fut enceint de moi

Sa jambe fut ma mère

Une ronce fut ma sage-femme

Un aigle m'emporta

Une vieille femme me trompa

Un beau prince m'emmena

Dans son palais il m'installa

Maintenant le fils embrasse la belle-mère

Et je garde les oies!

Naissance merveilleuse, enfant abandonné, chutes et montées vertigineuses, du ciel à la terre et aux enfers, et réciproquement, acquisition et perte de l'objet d'amour, retrouvailles. Passages du mythique à l'humain et réciproquement. L'agent transformateur est toujours la mauvaise mère. L'agent initiateur, c'est elle aussi. Serait-ce là alors l'une des voies énigmatiques qu'emprunte l'héroïne du conte merveilleux?

Je voudrais terminer en comparant les deux comptines mystérieuses, celle du conte-nouvelle et celle du conte merveilleux:

Pour le conte-nouvelle, le héros est autonome, sans aide magique. Le « monde autour de lui s'est aplati » selon l'expression de M. L. Tenèze (2000 : II-I2). Le créateur d'énigmes a donc dû voler son savoir à son propre destin. Le héros du conte-nouvelle advient comme sujet grâce à son expérience de passage obligé par la mort à la vie, grâce à sa nouvelle naissance de rescapé, sans avoir pourtant réalisé une descente « héroïque » aux Enfers. Il n'y a donc pas d'étages magiques, pas d'envolées au ciel, pas de chutes dans l'autre monde dans le conte-nouvelle. Le héros passe par la mort (et les morts) pour advenir à la vie, à sa propre vie, en empruntant un chemin aplati, dit « réaliste » cad, dépourvue de magie. Il s'invente des métaphores pour rendre compte de son passage initiatique. Il crée ainsi une énigme codée qui le rend désirable aux yeux de la princesse.

Pour le héros du conte merveilleux en revanche, le voyage comporte la découverte d'espaces sauvages où il va se faire initier. Il comporte des chutes et des montées vertigineuses, des virées dans d'autres mondes mythiques, des mondes à étages. Le héros voyageur peut être rattrapé là-bas par son entourage hostile, tomber amoureux ou être sauvé par ses aides magiques. Transformé en être ailé, il chante sur un arbre l'énigme qui met en récit sa propre histoire, sa fiction personnelle. Transformé en animal, du fond d'un puits, il chante toujours son histoire et il est reconnu par ceux qui l'aiment. Est-ce que l'énigme est toujours liée à une rencontre ou à des retrouvailles amoureuses? 
L'héroïne du conte merveilleux pour sa part, se présente lors de la séquence finale comme étant un conte elle même. N'oublions pas que par cette même occasion, le conteur en profite pour nous faire connaître son idée sur le conte merveilleux, sur le récit mythique de l'humanisation de l'enfant artificiel.

\section{Annexe}

\section{Ma mère fut une jambe}

Il était une fois un pope et sa femme qui n'avaient pas d'enfants, et leur jument était stérile, frappée par le même malheur que ses maîtres. Le prêtre, ouvertement et en cachette, priait dieu jour et nuit de leur donner un enfant pour leurs vieux jours. Il demandait aussi un poulain pour leur jument; mais il n'en fut rien; dieu n'écoutait pas ses prières. La femme du pope était malheureuse aussi. Elle faisait le tour de toutes les églises, en cachette, pour prier ; elle faisait des promesses de dons, et d'ex-voto en or et en argent; elle jeunait et accomplissait de bonnes œuvres de toute sorte, mais les années passaient sans qu'il y ait d'enfant pour eux. Le couple était désespéré. La femme du pope demandait à son mari de l'emmener chez le médecin; mais il ne voulait rien savoir, il lui disait: "Si dieu ne veut pas nous donner d'enfant, ni de poulain pour notre jument, cela veut dire qu'il s'agit réellement de sa volonté. Les humains ne sauraient être plus grands que dieu. »

Mais la femme du pope ne désespérait pas; un jour, une voisine lui dit: «J'ai appris, madame la pope, qu'il est arrivé dans un village d'à côté un magicien qui prescrit aux femmes stériles, ainsi qu'aux juments, des préparations à avaler. Elles se trouvent enceintes aussitôt après ». A l'entendre, la femme du pope eut le cour qui battait la chamade, elle voulut y aller aussitôt, mais elle ne savait quel prétexte fournir à son mari. Il aurait été entièrement opposé, elle en était sûre. Les deux femmes se mirent d'accord entre elles pour dire qu'elles allaient se rendre au village de la voisine dont elle était originaire; il y avait là bas une icône de la Sainte Vierge, réputée comme miraculeuse, car elle exauçait tous les vœux des pèlerins. Les deux femmes mentirent au pope, se préparèrent le soir, et partirent à l'aube le lendemain matin.

Arrivées au village, elles allèrent directement voir le sorcier. Une foule de jeunes femmes sans enfant attendaient alignées devant sa porte pour recevoir les préparations magiques; elles le payaient d'abord et le sorcier donnait à chacune ce qu'il lui fallait.

Enfin le tour de la femme du pope arriva. Elle lui raconta le chagrin qui la consumait, elle lui parla même de sa jument. Il l'écouta bien et dit : — N'aie pas peur, toi aussi tu auras un enfant, et ta jument aura un poulain. Voici cette pomme; tu la mangeras avant de te coucher et tu donneras les épluchures à la jument. Dans neuf mois, tu auras un enfant, dans onze mois, elle aura un poulain ». Folle de joie, la femme du pope lui paya tout ce qu'il avait demandé. Elle rentra dans son village avec son amie dont elle bénissait toute la lignée des ancêtres qui étaient aux cieux, pour l'avoir conduite auprès de ce magicien.

Elles arrivèrent au village le soir au coucher du soleil. Le pope l'attendait pour le dîner, il avait l'habitude de se coucher tôt, car il devait se réveiller pour les matines. — «Comment c'était, ma chère femme, au village? —-Très bien, mon pope. Il y avait beaucoup de femmes à l'église de la St. Vierge là bas, qui, comme moi, 
désiraient un enfant et faisaient le pèlerinage. —Que dieu puisse vous venir en aide, dit le pope bien tristement, car il se souvint qu'il désirait lui aussi un enfant. Viens manger, car tu es un peu en retard. -Je me prépare vite, c'est que nous avions assisté aux vêpres, dit la femme et partit cacher la pomme, qu'elle sortit discrètement du panier et posa sur une étagère. Elle la mangerait plus tard, juste avant de se coucher, en cachette de son mari. Après le dîner, le pope se mit à lire, comme tous les soirs, l'évangile du lendemain. La femme débarrassa, fit la vaisselle et prépara le lit en pensant à la pomme, quand on frappa à la porte. C'était une autre voisine qui l'appela pour lui tenir compagnie, car sa vieille mère était en train de mourir. Elle avait peur d'être seule et savait que la femme du pope était toujours prête à aider les gens. En effet, la femme du pope partit aussitôt chez la voisine pour l'assister en ce moment difficile.

Le pope finit sa lecture, ferma le livre, alla dans la cuisine boire un verre d'eau avant de se coucher. Tout d'un coup, il vit la pomme sur l'étagère.

-Quelle belle pomme, toute rouge! Je parie que ma femme me l'a apportée de l'autre village et qu'elle a oublié de me la donner.

Il prit la pomme, l'éplucha et la mangea en jetant les épluchures à la poubelle. Il se coucha sans plus attendre sa femme.

Elle arriva à minuit. Comme son mari était bien endormi, elle courût à la cuisine pour chercher la pomme, mais qui était introuvable.

«Mon dieu! Qu'ai-je fait! Le pope a dû la manger et jeter les épluchures ! Qu'est-ce que je vais devenir! Que faire, mon dieu ! En vain, je me suis donné tout ce mal! » Elle courut vite à la poubelle, prit les épluchures et les donna à la jument. «Fais-nous un petit poulain, toi, au moins, pour nous consoler un peu ». Elle comprima son chagrin, car elle comprit que c'était bien la volonté de dieu qu'elle reste sans enfant. Et le temps passait. Un jour, le prêtre remarqua soudain que l'une de ses jambes s'était mise à gonfler. Le mal empirait tous les jours; le pope décida alors de consulter un médecin en ville. Tous les deux pensèrent que c'était un rhumatisme. Entre temps, la jument se trouva pleine. L'on se réjouit, mais la pauvre femme qui savait ce qui s'était passé, avait le cour noir, d'avoir perdu un enfant de façon aussi injuste.

Un beau matin, le pope, souffrant, monta sur son âne et se mit en route pour la ville afin de soigner sa jambe enflée. Mais, sur le chemin, il eut tellement mal, qu'il descendit de l'âne et voulut s'allonger par terre sur une couverture qu'il posa sous un arbre, en attendant que sa douleur diminue; or, la douleur augmentait sans cesse et le pope finit par s'évanouir. Au moment même où il tombait par terre, une grosse épine, d'un buisson qui se trouvait là, lui déchira la peau de la jambe. Ainsi mit-il au monde une petite fille toute belle, un nouveau né merveilleux.

Pendant que le pope était encore endormi, alors qu'il avait toujours les yeux fermés, un aigle arriva. Il était à la recherche de nourriture; il vit le petit nourrisson de loin, fit un vol plané, le saisit et l'emporta là-haut dans son nid. Cet aigle était une femelle qui adopta la petite fille et l'éleva.

Quand le pope se réveilla, il vit sa jambe déchirée; le mal était parti; il ne comprit rien de ce qui s'était passé. «Dieu merci, se dit-il, je n'ai plus mal; l'abcès a dû être crevé, car voilà que ma jambe est déchirée! Je me sens très bien. Pourquoi donc aller en ville chez le médecin? Je vais plutôt rentrer à la maison, me faire soigner par ma femme! » Il entoura d'un bandage sa jambe blessée, monta sur son 
âne et s'achemina vers son village. Il rentra chez lui, mais il ne sut jamais, ni lui ni sa femme, que sa jambe avait donné naissance à une belle petite fille.

Laissons le prêtre et sa femme à présent et tournons-nous vers l'aigle et le nourrisson, la petite fillette. La dame aigle l'éleva comme sa propre fille. Elle lui apportait la meilleure nourriture, le lait même des oiseaux ; jusqu'à ce que la fillette devienne une jeune fille, jolie comme une poupée. Elle se promenait dans la forêt, montait sur les arbres et pensait à la façon dont elle s'était trouvée nourrisson endormi auprès d'un pope! Elle réfléchissait à son histoire que l'aigle lui avait racontée, car il parlait avec une voix humaine. Elle rencontrait de temps à autres des gens, qui venaient dans la forêt pour couper du bois, et quelques petites vieilles qui ramassaient des herbes et des brindilles pour l'hiver. Mais personne ne pouvait lui dire qui était ce pope et pourquoi elle s'était retrouvée avec l'aigle.

Un jour, un roi passait par là pour chasser avec ses douze ministres. Il la vit assise sur une branche de l'arbre et sa beauté le rendit fou. Il alla sous l'arbre et lui dit:

-Viens avec nous, la belle, et je te donnerai tout ce que tu voudras! - Je ne descends pas, je ne fréquente pas les humains, car je suis les conseils de ma mère l'aigle! —Et que te dit ta mère l'aigle? —Que les humains sont méchants —Il ne faut pas la croire, descends pour qu'on en parle et tu verras qu'elle te ment! —Si je descends, tu me caresseras peut-être! —Bien sûr, je te caresserai, si tu descends! —Voilà, tu l'admets toi-même! Ma mère me dit que les mains humaines font toujours mal, même lorsqu'elles prodiguent des caresses!

La belle jeune fille resta assise sur son arbre. Mais le roi, fou amoureux, allait tous les jours la chercher dans la forêt. Elle le voyait venir de loin; son cœur lui disait de s'approcher; mais elle suivait les conseils de sa mère l'aigle et, apeurée, elle grimpait toujours sur l'arbre. Un jour, le roi rencontra sur son chemin une petite vieille qui ramassait des brindilles. - Ma petite dame, est-ce que tu connais une jeune fille qui vit sur les arbres? —-Tu veux dire, mon seigneur, la fille de l'aigle? Non seulement je la connais, mais elle est mon amie. Elle a comme seul parent un aigle qui l'a élevée en prenant soin d'elle comme de la prunelle de ses yeux, il lui apportait le lait même des oiseaux pour la nourrir; il la berçait sous ses ailes! —Et comment puis-je faire pour la convaincre de descendre de l'arbre? Je lui promets plein de choses, je fais tout mon possible, sans résultat! —Ne t'en soucie guerre, je vais te la faire descendre. Viens demain avec ton cheval, mais cache-toi dans un coin et sois prêt à la saisir et à l'enlever.

Le lendemain, la vieille alla s'asseoir sous l'arbre. Le roi était caché derrière un buisson avec son cheval. La jeune fille parlait assise sur sa branche comme tous les jours avec la vieille femme. Celle-ci ouvrit sa besace, prit quelques champignons, les lava, puis elle alluma un feu et se mit à les frire en versant de l'huile sur une poêle. Mais elle tenait la poêle à l'envers et versait l'huile sur le feu qui flambait de plus en plus. La fille riait en la voyant faire et disait: - Mais enfin, tu ne vois pas que la poêle est à l'envers? Tu jettes l'huile sur le feu et ça flambe! —Je ne vois pas très bien, mon enfant! A l'envers, tu dis? Comment ça? Descends pour me montrer comment faire. J'ai voulu, pour une fois faire frire des champignons et voilà que je ne sais comment faire!

La jeune fille descendit de l'arbre, mais, à peine eut-elle mit le pied par terre, le roi la saisit, l'emporta sur son cheval qu'il éperonna; en galopant bride abattue, il fonça jusqu'au palais. Arrivés là bas, toute la cour sortit pour admirer la 
belle jeune fille que le roi avait amenée. Il ordonna qu'on l'habille avec des habits royaux et, quelques jours plus tard, il l'épousa.

Ils vivaient très heureux en riant toujours et en s'amusant. Mais la méchante marâtre du roi, - qui n'était pas très veille - était jalouse de la beauté de sa bru et voulait s'en débarrasser. Elle aurait voulu épouser elle-même son beau-fils afin de rester reine pour toujours. Le pauvre roi n'avait pas idée que sa belle-mère pensait de la sorte. Et la marâtre cachait bien sa jalousie en attendant l'occasion pour détruire la jeune fille. Or, voilà que l'occasion se présenta de façon inopinée.

Un pays voisin leur déclara la guerre. Depuis l'époque de son père, celui-ci avait toujours été leur ennemi. Toutes les provinces furent sur le pied de guerre, le roi prépara son armée pour combattre l'ennemi. Il dit alors à sa belle-mère : -Maman, (il l'appelait ainsi, ne pouvant pas connaitre ses arrières pensées) je dois m'en aller; je te confie ma femme pour que tu prennes soin d'elle, car elle est seule dans le monde, sans famille. - Ne t'inquiète pas! Tu peux partir tranquille; je m'occuperai d'elle comme d'une sœur, elle ne manquera de rien. Le roi la crut et se réjouit. Il fit ses adieux aux deux femmes et s'en alla.

La jeune femme s'enferma dans sa chambre et pleura toutes les larmes de son corps; elle ne voulait voir personne. Elle se souvenait de son aigle et pleurait d'autant plus. Quinze jours plus tard, lorsque la marâtre fut assurée que le roi était déjà assez loin, elle convoqua sa bru et lui dit: — « Toi, fille d'aigle, sans famille aucune, tu n'es pas faite pour être reine et femme de roi ! Car la reine c'est moi ! Ne crois pas que je suis vieille. Si mon beau fils ne t'avait pas trouvée dans les montagnes, il m'aurait épousée moi. Tu es arrivée inopinément et tu me l'as enlevé, mais, ne t'en fais pas, moi je t'aurai. Si tu veux rester en vie, tu dois disparaître d'ici, sinon je te tue! Tu iras garder les oies loin, très loin, aux confins de nos terres. Et tu ne dois en parler à personne; si tu dis le moindre mot à quelqu'un, tu perdras ta tête. Je le saurai immédiatement, car l'un de mes serviteurs, le bourreau du palais, serra toujours à tes trousses, il te guettera jour et nuit. Tu disposes d'un délai de deux jours pour t'en aller!».

La jeune femme, confuse, se mit à trembler de peur. Elle se souvint des paroles de l'aigle à propos des humains, mais trop tard. Le lendemain matin, avant l'aube, pour que personne ne la voit, elle quitta le palais habillée comme une pauvresse et alla garder les oies sur des terres qui lui avaient appartenues jusqu'au jour précédent; elle se mit à faire picorer les oies.

Trois ans s'écoulèrent. La guerre était finie; elle apprit par d'autres serviteurs que le roi allait rentrer. Mais elle n'avait plus aucun espoir de le revoir.

Le roi revint désireux de retrouver sa femme bien-aimée, qu'il n'avait pas vue depuis trois ans. Il n'avait plus de ses nouvelles. Mais, il ne vit que sa belle-mère qui l'accueillit au palais. —Où est ma femme? demanda-t-il, inquiet. —Ta femme bien-aimée a quitté le château dès que tu as eu le dos tourné, elle est partie retrouver son aigle. De toi, elle n'a jamais parlé! Le roi fut rempli de chagrin; il s'enferma dans sa chambre et pleura beaucoup, car il aimait sa femme, cour et âme. La bellemère ne savait pas comment l'amadouer. Néanmoins, elle était devenue la reine du pays, c'était elle qui gouvernait. Elle faisait ce que bon lui semblait.

Le roi ne désirait plus rien faire. Il sortait seulement la nuit, il se promenait tout seul dans l'obscurité, sans que personne ne le voie. Il ne pouvait pas croire que sa femme, qu'il aimait tant, l'avait quitté pour retrouver son aigle. Un oiseau! 
Une nuit, alors que, comme d'habitude, il ne dormait pas, il sortit se promener dans son domaine pour prendre l'air. Sans faire attention, il s'approcha d'une petite maison au bout d'un terrain, qui était le domicile des serviteurs. Dans la tranquillité de la nuit, il entendit résonner des rires et des palabres. Il se dit: Voyons voir de plus près ce que mes domestiques se disent, car ils ont l'air beaucoup plus joyeux que moi! Il s'approcha donc et se cacha tout près d'une fenêtre pour mieux écouter. C'était l'heure des contes. Le roi aimait les contes; il s'assit pour écouter. Alors, une femme se mit à crier: - Viens ici, la gardeuse d'oies! Raconte-nous aussi un conte! Tu ne parles jamais; tu restes toujours cachée dans la pénombre et tu pleures. Raconte-nous, que veux-tu? Quel est le chagrin qui te consume? -Oui, oui, elle a raison, lui crièrent alors d'autres voix. Viens ici, devant nous tous, et raconte-nous, toi aussi, un tout petit conte!

Le roi entendit alors une voix qu'il connaissait, qui le fit frémir. —Il me semble la reconnaître cette voix, se dit-il, et tendit mieux l'oreille. Il entendit alors la gardeuse d'oies, qui pendant trois ans n'avait parlé à personne. Depuis sa place dans l'ombre, elle se mit à chanter:

Un conte à raconter?

Conte je suis!

Une femme de prêtre me désira

Une pomme me conçut

Un prêtre fut enceint de moi

Sa jambe fut ma mère

Une ronce fut ma sage-femme

Un aigle m'emporta

Une vieille femme me trompa

Un beau prince m'emmena

Dans son palais il m'installa

Maintenant le fils embrasse la belle-mère

Et je garde les oies!

—Quel beau conte! Et si curieux! Tous les serviteurs lui crièrent « encore, encore un fois », en l'applaudissant. Ils riaient de tout cœur avec les dires de la gardeuse d'oies, qui avait inventé une si belle histoire. - Voyez donc ça, la gardeuse d'oies qui ne parlait jamais! Répète-nous ce conte, il est plein de mystère!

La pauvre femme répéta encore deux fois son histoire avec la voix tremblante. A ce moment, la porte de la maison s'ouvrit et le roi leur apparut. A le voir ainsi, ils furent tous ébahis et tombèrent à ses pieds pour témoigner leur respect. Il dit alors:

—N'ayez pas peur! Ne bougez pas! Je veux juste voir celle qui a récité ce conte. Qui est-elle? —La gardeuse d'oies! La gardeuse d'oies! lui crièrent alors tous ensemble en battant leurs petits tambourins. Apeurée, elle s'était cachée dans le coin le plus obscur, mais ils la prirent par la main et l'obligèrent à s'approcher du roi. Il la reconnut aussitôt, la prit dans ses bras et lui dit: —C'est toi, ma femme bien aimée? Tu n'es pas retournée dans ton nid d'aigle? —Quel nid d'aigle? Ta marâtre m'a chassée, elle m'a envoyé garder les oies, car elle était jalouse de notre 
amour. Elle voulait te prendre pour époux, afin de toujours régner ! -Elle m'a dont menti de cette façon éhontée? Quelle femme jalouse et perverse ! Je vais ordonner qu'on l'exécute!-Mais, non, je ne veux pas que tu la tues, puisque nous sommes à présent réunis tous les deux! - Alors, je la jetterai dans un cachot obscur pour le restant de ses jours.

Voilà comment la gardeuse d'oies redevint la reine du pays et régna dans toute sa beauté. Et, lorsque tout fut redevenu calme dans le palais, la reine demanda une faveur à son époux, d'aller voir l'aigle qui l'avait élevée. Un beau matin, ils montèrent à cheval et partirent vers la montagne de la dame aigle. Ils la retrouvèrent vieille et malade. Ils l'emmenèrent alors avec eux au palais. Sur l'ordre de la reine, on fit construire un très beau nid pour que la mère aigle puisse résider et passer le restant de sa vieillesse paisiblement comme elle le méritait. Car elle l'avait si bien élevée pendant de longues années!

Ainsi vécurent-ils heureux et nous encore mieux.

Stella EPIFANIOU-PÉTrAKI (I968): Contes de Smyrne, III. Athènes.

\section{L'énigme du paresseux}

Il était une fois une mère qui avait un fils, si paresseux qu'elle voulait se débarrasser de lui.

Un beau jour, elle le mit à la porte de la maison: «va trouver de quoi vivre! » lui intima-t-elle. Et elle lui donna une pita pour la route. Une pita empoisonnée. La mère donna aussi à son fils une petite chienne, du nom de Morfoula, pour qu'elle lui tînt compagnie.

Le fils se mit en route. En chemin, il s'arrêta pour manger : il ouvrit son baluchon et se coupa une part de la pita. Il s'apprêtait à l'avaler mais dieu voulut d'abord qu'il en offrît un morceau à sa petite chienne. Et à la première bouchée, Morfoula mourut. Voyant cela, le jeune homme songea à sa mère et au sort qu'elle lui avait préparé.

C'est alors qu'un aigle passa dans le ciel, qui fondit sur la chienne pour la dévorer. Le jeune homme lui décocha des flèches pour le repousser; l'aigle s'envola, emportant sa proie et les flèches du jeune homme atteignirent une vache qui broutait l'herbe du pré voisin. Le jeune homme, qui mourrait de faim, voulut manger l'animal et il s'approcha de la vache pour la dépecer. Il vit alors surgir un tout petit veau du ventre de la bête, qui sautillait gaiement. Le garçon découpa la vache en morceaux et se prépara des côtelettes. Seulement, il lui manquait le feu! Il regarda autour de lui et vit une petite église: à l'intérieur, se trouvaient des livres, qu'il fit brûler; sur le feu, il fit griller ses côtelettes. Lorsqu'il les eut mangées, il voulut boire. Point de fontaine dans les environs: il but l'eau du bénitier.

Puis il quitta l'église et se remit en route. Une nouvelle chose se produisit encore sous ses yeux : un troupeau de chèvres, qui descendait de la colline et qui s'était engagé sur le pont pour traverser la rivière, fut emporté par un énorme torrent; toutes les chèvres périrent, le pont fut détruit.

Le garçon reprit sa route. Quelques temps plus tard, il parvint au palais d'un roi qui avait naguère fait une annonce : celui qui lui poserait une énigme insoluble, qui ne soit pas prise d'un livre, avait-il dit, gagnerait la main de sa fille. Mais 
s'il advenait que l'énigme était d'un livre, alors on couperait la tête de celui qui l'aurait posée.

Le jeune homme se présenta au palais, habillé de haillons et pieds nus. "Que veux-tu? » lui demanda-t-on — « présenter mon énigme au roi ! » répondit-il. Et tout le palais éclata de rire. Mais le roi qui avait entendu le jeune homme ordonna qu'on le fît monter. Alors, le jeune homme énonça son énigme, qui était sa propre histoire, ainsi qu'il l'avait vécue:

La pita tua Morfoula

Un être volant passa et attrapa Morfoula

Je lance des flèches où mon œil les porte, je frappe un être né

Et je libère un être qui n'était pas né!

Je mange un rôti, cuit par des écritures!

Je bois de l'eau,

ni de la terre, ni du ciel!

Le faible qui bouge l'emporte sur le fort qui reste immobile!

Cette énigme n'avait pas été trouvée dans un livre; tous furent admiratifs, le roi donna sa fille en mariage et le conte est terminé!

\section{Références bibliographiques}

AmAdes, Joan (I960): «Les contes-devinettes de Catalogne ». Fabula vol. 3, nº 2 (I960): I99-223.

Chardenet, Virginie (20Io) : Destins de garçons en marge du symbolique. Paris : Corti Les essais merveilleux.

Delarue, Paul (†); Marie-Louise Tenèze (2000): Le conte populaire français. Contesnouvelles. Paris: Editions du Comité des travaux historiques et scientifiques.

Megas, Georgios A. $(\dagger)$; Anna Angelopoulos; Aigli BrousKou ; Marianthi KAPLANOGLOU ; Emmanouella KATRINAKI (2OI2) : Catalogue of Greek Magic Folktales. Folklore Fellows' Communications 303. Helsinki : Suomalainen Tiedeakatemia.

UTHER, Hans-Jörg (2004) : The Types of International Folktales. Folklore Fellows' Communications 284-285-286. Helsinki: Suomalainen Tiedeakatemia. 